\title{
Acknowledgement to Reviewers of Big Data and Cognitive Computing in 2018
}

\author{
Big Data and Cognitive Computing Editorial Office \\ MDPI, St. Alban-Anlage 66, 4052 Basel, Switzerland \\ Published: 21 January 2019
}

\begin{abstract}
Rigorous peer-review is the corner-stone of high-quality academic publishing. The editorial team greatly appreciates the reviewers who contributed their knowledge and expertise to the journal's editorial process over the past 12 months. In 2018, a total of 40 papers were published in the journal, with a median time to first decision of 13 days and a median time to publication of 47 days. The editors would like to express their sincere gratitude to the following reviewers for their cooperation and dedication in 2018:
\end{abstract}

\begin{tabular}{|c|c|}
\hline Almgren, Khaled & Fabbrocino, Giovanni \\
\hline Aloqaily, Moayad & Fiorentino, Michele \\
\hline Ariyur, Kartik & Fraga-Lamas, Paula \\
\hline Baker, Thar & Gao, Longxiang \\
\hline Bauberger, Stefan & García-Díaz, Pilar \\
\hline Baum, Seth & García-Gonzalo, Esperanza \\
\hline Beganovic, Nejra & Garg, Harish \\
\hline Behzadan, Vahid & Gatteschi, Valentina \\
\hline Benninger, Daniel & Geng, Ruili \\
\hline Bernardino, Jorge & Gibson, David \\
\hline Bölöni, Ladislau & González Pérez, Isaías \\
\hline Boussaid, Omar & Grivokostopoulou, Foteini \\
\hline Bujnowski, Adam & Guo, Xinfei \\
\hline Caleffi, Marcello & Hao, Yixue \\
\hline Cao, Hung & Hedayatifar, Leila \\
\hline Carbonell Carrera, Carlos & Hemmati, Farzad \\
\hline Cerro, Gianni & Hölbl, Marko \\
\hline Chen, Liang-Bi & Huh, Jun-Ho Huh \\
\hline Corsar, David & Hussain, Zahir \\
\hline Cotfas, Liviu-Adrian & Infantino, Ignazio \\
\hline Cottafava, Dario & Iuppa, Claudio \\
\hline Daniel, Ben Kei & Jajoo, Aarti \\
\hline Demertzis, Konstantinos & Ji, Hao \\
\hline Dreżewski, Rafał & Ji, Shuiwang \\
\hline Dubey, Harishchandra & Johansen, Mikkel Willum \\
\hline Estêvão, João M.C. & Kaabouch, Naima \\
\hline Etemad, Katayoon & Kanavos, Andreas \\
\hline
\end{tabular}


Kang, James

Karakatič, Sašo

Koerich, Alessandro

Kotsiantis, Sotiris

Kouziokas, Georgios N.

Krasoulis, Agamemnon

Krygier, Jaroslaw

Le, Hieu Hanh

Lemnaru, Camelia

Li, Xiang

Lian, Wenzhao

Lin, Feng-Cheng

Livieris, Ioannis

Long, $\mathrm{Xi}$

Łopatka, Jerzy

Maestre, Jorge

Markakis, Evangelos K.

Martino, Luca

Mašek, Pavel

Masłowski, Andrzej

Mateos, Cristian

Mathapathi, Shivakumar

Mavragani, Amaryllis

Menon, Varun G.

Miao, Yiming

Minervini, Pasquale

Modoni, Gianfranco

Mori, Ryota

Moroni, Davide

Mousas, Christos

Mozgovoy, Maxim

Murtagh, Fionn

Necula, Sabina

Nguyen, Binh P.

Ojha, Varun Kumar

Oliveira, Teresa A.

Ovalle, María Antonia

Pagge, Jenny

Paiva, Sara

Pang, Jun

Pecori, Riccardo

Peimankar, Abdolrahman

Perikos, Isidoros
Petrosanu, Dana-Mihaela

Pozza, Riccardo

Rajaraman, Sivaramakrishnan

Reis, Catarina I.

Relich, Marcin

Rinaldi, Stefano

Rodríguez, Alfonso

Rosenbaum, Howard

Rybarczyk, Yves

Salazar, Addisson

Sanwar Hosen, A. S. M.

Savaglio, Claudio

Schumacher, Jens

Sicilia, Miguel-Angel

Smeaton, Alan

So, Chaehan

Song, Jeungeun

Sotala, Kaj

Sperlì, Giancarlo

Štajduhar, Ivan

Straub, Jeremy

$\mathrm{Su}$, Ming-Shou

Sun, Ziheng

Taffese, Woubishet Zewdu

Tamura, Hiroki

Tang, Wenwu

Teisseire, Maguelonne

Theron, Roberto

Tomaszuk, Dominik

Tonacci, Alessandro

Tramontana, Emiliano

Trovati, Marcello

Tsinos, Christos

Tsiropoulou, Eirini Eleni

Turchin, Alexey

Ullah, AMM Sharif

Ungureanu, Florina

Vahedian, Amin

Vaiciukynas, Evaldas

Van Den Noort, Maurits

Van Leeuwen, Tessa M.

Vinagre, Eugénia

Vlad, Diaconita 


\author{
Vlahu-Gjorgievska, Elena \\ Wang, Cheng \\ Wang, Jiayin \\ Wang, Junye \\ Weber, Felix \\ Wielgosz, Maciej \\ $\mathrm{Xu}$, Quanqing \\ Yang, Chaowei
}

\author{
Yu, Yang \\ Yue, Jing \\ Zhang, Ce \\ Zhang, Le \\ Zhang, Yudong \\ Zhao, Dongfang \\ Zhe, Li \\ Ziemba, Paweł
}

(C) 2019 by the authors. Licensee MDPI, Basel, Switzerland. This article is an open access article distributed under the terms and conditions of the Creative Commons Attribution (CC BY) license (http://creativecommons.org/licenses/by/4.0/). 